\title{
Rapid Identification of Psychoactive Drugs in Drained Gastric Lavage Fluid and Whole Blood Specimens of Drug Overdose Patients Using Ambient Mass Spectrometry
}

\author{
Chi-Wei Lee ${ }^{1,2}$, Hung Su${ }^{3}$, You-Da Cai ${ }^{3}$, Ming-Tsang $\mathrm{Wu}^{4}$, \\ Den-Chyang $\mathrm{Wu}^{5}$, and Jentaie Shiea*,3,6 \\ ${ }^{1}$ Graduate Institute of Medicine, Kaohsiung Medical University, Kaohsiung, Taiwan \\ ${ }^{2}$ Department of Emergency Medicine, Kaohsiung Medical University Hospital, Kaohsiung Medical University, Kaohsiung, Taiwan \\ ${ }^{3}$ Department of Chemistry, National Sun Yat-Sen University, Kaohsiung, Taiwan \\ ${ }^{4}$ Department of Public Health, College of Health Sciences, Kaohsiung Medical University, Kaohsiung, Taiwan \\ ${ }^{5}$ Division of Gastroenterology, Department of Internal Medicine, Kaohsiung Medical University Hospital, Kaohsiung, Taiwan \\ ${ }^{6}$ Department of Medicinal and Applied Chemistry, Kaohsiung Medical University, Kaohsiung, Taiwan
}

\begin{abstract}
Psychoactive drug overdoses are life-threatening and require prompt and proper treatment in the emergency room to minimize morbidity and mortality. Prompt identification of the ingested psychoactive drugs is challenging, since witness recall is unreliable and patients' symptoms do not necessarily explain their loss of consciousness. Gas and liquid chromatography mass spectrometric analyses have been the traditionally employed methods to detect and identify abused substances; however, these techniques are time-consuming and labor-intensive. In this study, thermal desorption electrospray ionization mass spectrometry, an ambient mass spectrometric technique, was applied to rapidly characterize flunitrazepam, lysergic acid diethylamide, and 3,4-methylenedioxy-methamphetamine in drained gastric lavage fluid, and ketamine, cocaine, amphetamine and norketamine in whole blood samples. No pretreatment of the gastric lavage fluid specimens was required and the entire analytical process took less than $30 \mathrm{~s}$ per specimen. Liquid-liquid extraction, followed by centrifugation, was performed on the whole blood samples. The corresponding compounds were identified through matching the obtained mass spectrometric data with those provided by commercial databases. The limits-of-detection of the tested drugs in both drained gastric lavage fluid and whole blood samples are at sub ppm levels. This is sensitive enough for emergency medical application, since the quantities of medications ingested by overdosed abusers are much higher than the amounts that were tested.
\end{abstract}

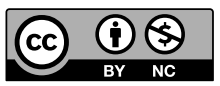

Copyright (c) 2017 Chi-Wei Lee, Hung Su, You-Da Cai, Ming-Tsang Wu, Den-Chyang Wu, and Jentaie Shiea. This is an open access article distributed under the terms of Creative Commons Attribution License, which permits use, distribution, and reproduction in any medium, provided the original work is properly cited and is not used for commercial purposes.

Please cite this article as: Mass Spectrom (Tokyo) 2017; 6(2): S0056

Keywords: emergency room, point-of-care identification, psychoactive drugs, biofluid, ambient mass spectrometry

(Received November 1, 2016; Accepted December 20, 2016)

\section{INTRODUCTION}

Drug abuse is heavily linked with issues such as robbery, theft, family disintegration, loss of employment, domestic violence, and child abuse. The monetary consequences of drug abuse in the United States, including productivity, health, and crime related costs, are estimated to exceed \$600 billion annually. ${ }^{1)}$ The World Health Organization acknowledges that at least 15.3 million persons have drug abuse-related disorders. Since drug overdoses are life- threatening, it is important to promptly and accurately identify substances consumed by emergency room patients.

Psychoactive drugs cause intoxication through various mechanisms that require different therapeutic strategies, precautions against contraindicating actions, directions of clinical course monitoring, and predictions of prognoses. For example, amphetamine and its derivatives are potent central nervous system stimulants, whilst opioid drugs are potent central nervous system depressants; treatment of amphetamine and its derivative intoxications is thus totally different from that of opioids. ${ }^{2,3)}$

\footnotetext{
*Correspondence to: Jentaie Shiea, Department of Chemistry, National Sun Yat-Sen University, Kaohsiung, Taiwan, e-mail: jetea@faculty.nsysu.edu.tw
} 
Identification of abused drugs is currently based on the history of exposure, clinical signs and symptoms, and laboratory measurements. Unfortunately, it is difficult to rapidly identify drugs taken by overdose patients in the early stages of emergency care, as most patients are unconscious and acute overdose toxidromes occur only after certain amount of drugs have been absorbed. In addition, not all signs and symptoms of psychoactive drug overdoses are present in every patient; the drugs have multiple pharmacologic functions and affect the brain in a variety of ways. ${ }^{4}$ Although immunoassay techniques have been introduced to identify psychoactive drugs in saliva, the narrow specificity of determination due to the variable cross-reactivities of the drugs with antibodies remains the biggest limitation of such immunochemical methods.5) Traditionally, abused drugs present in urine or blood are characterized by gas chromatography/mass spectrometry (GC/MS) and/or liquid chromatography/mass spectrometry (LC/MS) ${ }^{5,6)}$ Although both techniques are sensitive and reliable, laborious and time-consuming sample pretreatment processes such as solvent extraction, filtration, concentration, fractionation, and derivatization are needed to avoid interference from the predominant compounds in biological fluids. These conventional methods require hours for drug identification; thus, laboratory reports are not prompt enough to be used as clinical references for drug overdose patients in the emergency room.

Ambient mass spectrometry (AMS), a recently developed method, requires minimum or no sample pretreatment and dramatically reduces analytical time from hours to seconds. Thermal desorption-electrospray ionization/ mass spectrometry (TD-ESI/MS) is an AMS technique that characterizes trace chemical compounds in liquids, syrups, ointments, and solid states without undergoing extraction or separation. ${ }^{78)}$ In TD-ESI/MS, analytes such as residual pesticides on fruits and vegetables are collected by sweeping a metallic probe across the sample surface. Analytes on the probe are quickly desorbed in a preheated oven. The thermally desorbed analytes are subsequently carried by a nitrogen stream into an electrospray plume and ionized via ion-molecule reactions with the charged solvent species to form analyte ions. Even though sampling, desorption, ionization, and detection are separate events, a typical TD-ESI/ MS analysis takes less than $30 \mathrm{~s}$. Compared to other ambient ionization sources such as desorption electrospray ionization (DESI), ${ }^{9-11)}$ direct analysis in real time (DART), ${ }^{12,13)}$ and electrospray-assisted laser desorption ionization (ELDI), ${ }^{14)}$ where samples must meet the ion source, the use of a direct sampling probe in TD-ESI/MS makes the technique suitable to analyze pesticides and drugs on oversized or immovable objects and in viscous biological solutions. ${ }^{7,8,15,16)}$

During early rescue, gastric lavage is a common practice for removing sustained-release medications and ingested substances. ${ }^{17)}$ To promptly identify the ingested compounds, residual psychoactive drugs in the drained gastric fluid should also undergo drug identification analysis. In this study, we developed point-of-care analytical strategies using TD-ESI/MS to rapidly identify commonly abused drugs consumed orally and found in drained gastric lavage fluid (flunitrazepam, lysergic acid diethylamide, and 3,4-methylenedioxy-methamphetamine) and parenterally in blood (ketamine, cocaine, and amphetamine, and norketamine). This approach is capable of expediting emergency medical care and provides important toxicological information for decision-making during critical resuscitations.

\section{EXPERIMENTAL}

\section{Reagents}

The standards of six commonly abused drugs in Taiwan were obtained from Sigma-Aldrich (St. Louis, USA), including flunitrazepam (FM2), lysergic acid diethylamide (LSD), 3,4-methylenedioxy-methamphetamine (MDMA), ketamine, cocaine, amphetamine and one of its metabolites-norket-
Central Nervous System Depressants

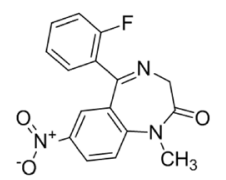

flunitrazepam, FM2

$\mathrm{C}_{16} \mathrm{H}_{12} \mathrm{FN}_{3} \mathrm{O}_{3}$

$[\mathrm{M}+\mathrm{H}]^{+}=314$

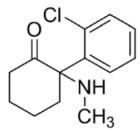

ketamine

$\mathrm{C}_{13} \mathrm{H}_{16} \mathrm{ClNO}$

$[\mathrm{M}+\mathrm{H}]^{+}=238$

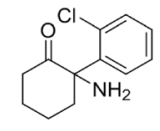

norketamine

$\mathrm{C}_{12} \mathrm{H}_{14} \mathrm{ClNO}$

$[\mathrm{M}+\mathrm{H}]^{+}=224$
Hallucinogen

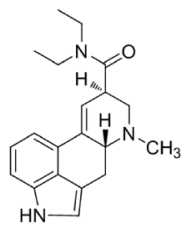

lysergic acid diethylamide, LSD

$\mathrm{C}_{20} \mathrm{H}_{25} \mathrm{~N}_{3} \mathrm{O}$

$[\mathrm{M}+\mathrm{H}]^{+}=324$

\section{Central Nervous System Stimulants}

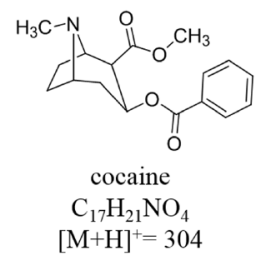

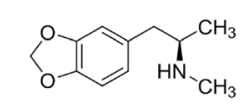

3,4-methylenedioxymethamphetamine, MDMA $\mathrm{C}_{11} \mathrm{H}_{15} \mathrm{NO}_{2}$ $[\mathrm{M}+\mathrm{H}]^{+}=194$

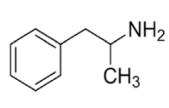

amphetamine

$\mathrm{C}_{9} \mathrm{H}_{13} \mathrm{~N}$

$[\mathrm{M}+\mathrm{H}]^{+}=136$

Fig. 1. All chemical structures, formulas, and $\mathrm{m} / \mathrm{z}$ values of drugs, including a metabolite of ketamine (norketamine), tested in this study. 
amine. They were used without further purification. Figure 1 shows the structures, chemical formulas and $\mathrm{m} / \mathrm{z}$ values of the drugs. To simulate clinical conditions, these recreational drugs that are often consumed orally (FM2, LSD, and MDMA) were spiked in drained gastric lavage fluid, while those consumed parenterally (ketamine, cocaine, amphetamine, and norketamine) were spiked in whole blood.

Solvents for electrospray ionization (i.e., methanol and acetic acid) were purchased from Merck (Darmstadt, Germany) and Sigma-Aldrich, respectively. Distilled deionized water (purified with a PURELAB Classic UV from ELGA, Marlow, UK) was used to prepare the electrospray solution. HPLC-grade solvents such as dichloromethane (DCM), hexane, toluene, and ethyl acetate (EA) were obtained from J.T. Baker (Phillipsburg, USA) and used for liquid-liquid drug extraction.

\section{Sample collection}

Gastric juice specimens ( $15 \mathrm{~mL}$ each) were collected from two volunteers who received routine gastroendoscopic examinations. The volunteers' informed consents were obtained under the approval of the institutional review board of Kaohsiung Medical University Hospital (KMUHIRB-20130004). The gastric juice specimens were stored under $-80^{\circ} \mathrm{C}$, and stock solutions were prepared by diluting each of the drug standard solutions with distilled deionized water to $1000 \mathrm{ppm}$. For TD-ESI/MS measurements, the samples were obtained by diluting stock solutions (from $1000 \mathrm{ppm}$ to $1 \mathrm{ppb}$ ) with diluted gastric juice.

The collection of whole blood specimens was carried out through venipuncture after informed consent of a volunteer under the approval of the institutional review board of Kaohsiung Medical University Hospital (KMUHIRB-20130004). Five milliliters of whole blood were drawn and collected in a collection tube with heparin, frozen, and then stored. The whole blood specimens were allowed to stand at $25^{\circ} \mathrm{C}$ for $10 \mathrm{~min}$ prior to further analysis. They were spiked with drugs, then mixed with organic solvent and homogenized by gentle vortexing for $20 \mathrm{~s}$. The mixture was centrifuged for $2 \mathrm{~min}$ at $12000 \mathrm{rpm}$, and the supernatant (organic phase) was transferred to an eppendorf. For quantitative analysis, the supernatant was then evaporated under nitrogen. The dry residue was reconstituted in $100 \mu \mathrm{L}$ of organic solvent for further analysis.

\section{Mass spectrometric analysis}

The TD-ESI/MS technique was set up in a similar manner as described in previous publications. ${ }^{7,8)}$ In short, a TD-ESI source comprised of a metallic sampling probe (60 $\mathrm{mm}$ long, $2.5 \mathrm{~mm}$ in diameter; Ming Yuh Scientific Instruments Co., Tainan, Taiwan), thermal desorption unit, and electrospray ionization interface were coupled to a triple quadrupole mass spectrometer (Agilent 6410B, Santa Clara, CA, USA) for MS and MS/MS analyses. The sampling probe was dipped and removed quickly from the sample solution (drained gastric lavage fluid or organic solvent), and then inserted promptly into the TD-ESI source. In order to remove memory effects from previous samples, the tip of the probe was burnt with a high-temperature flame from a hand-held gas torch. The desorption temperature of the TD-ESI source was set at $280^{\circ} \mathrm{C}$ using a temperature controller (ANLY AT-502, Taipei, Taiwan). A metal tubing was attached to the heated oven in order to carry the heated nitrogen gas prior to entering the desorption area. The nitrogen gas (5 psi) entered the top of the TD unit and carried the desorbed analytes into the ESI plume. The electrospray solution was comprised of methanol, water, and acetic acid (50/50/0.1 by volume). A high voltage $(4.5 \mathrm{kV})$ was applied to the ESI capillary to induce electrospray ionization via solution conduction. The entire experimental procedure is shown in Fig. 2. (a) Sample collection

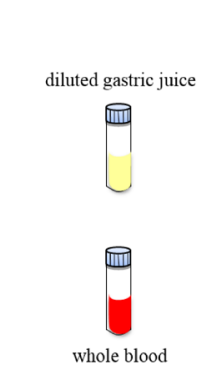

(e) Resuscitation

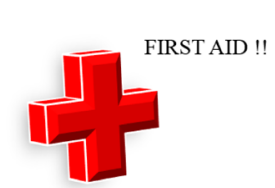

(b) Sampling solution with a probe

(c) TD-ESI/MS analysis
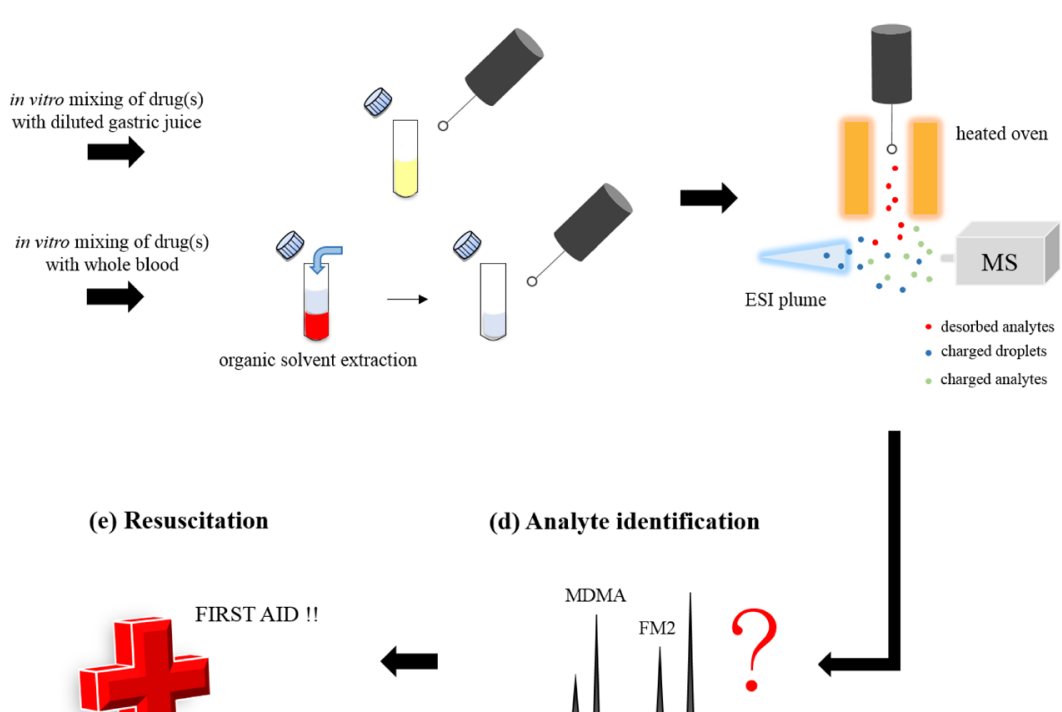

(d) Analyte identification

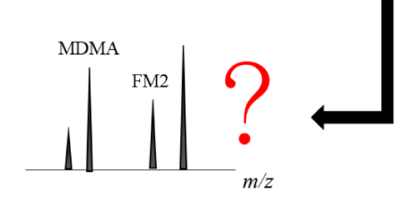

Fig. 2. Analytical processes for characterizing drugs in biofluids: (a) drugs were spiked in drained gastric lavage fluid and whole blood; (b) a metallic probe was used to transfer the sample solutions with or without solvent extraction; (c) analytes were thermally desorbed from the probe and ionized in an electrospray plume in a TD-ESI source; (d) mass spectral data of the corresponding drugs were acquired, matched to drug databases, and the intoxicants identified; and (e) in an emergency room situation, the data obtained could allow for a prompt response initiated within one minute. 


\section{Mass spectral database}

Once mass spectra of the abused drugs were obtained by means of TD-ESI/MS and TD-ESI/MS/MS analyses, the mass spectrometric data (such as $\mathrm{m} / \mathrm{z}$ values of precursor and product ions of analytes) were uploaded to the as-built software-MassHunter Veterinary Drug Triggered MRM Database and Library (Agilent Technologies) for matching and identifying the compound (more information can be seen in the following guideline, page from 12 to $43 \mathrm{http}: / /$ www.ingenieria-analitica.com/downloads/dl/file/id/2984/ product/110/masshunter_veterinary_drug_triggered_mrm_ database_and_library_quick_start_guide.pdf).

\section{RESULTS AND DISCUSSION}

In the absence of patient's history of drug abuse, diagnosis of psychoactive drug overdoses in drug abusers in emergency room is usually made by inefficiently indirect inference. Since TD-ESI/MS has been demonstrated to rapidly characterize residual pesticides and oral medications in biofluids, ${ }^{15,16)}$ the technique was used in this study to detect commonly abused drug standards. It was found that the protonated molecular ions $\left(\mathrm{MH}^{+}, \mathrm{M}\right.$ : analyte) for six drugs and one of their metabolites were all detected: FM2 ( $m / z$ 313), LSD $(m / z$ 324), MDMA ( $m / z$ 194), ketamine $(\mathrm{m} / \mathrm{z} 238)$, cocaine $(\mathrm{m} / \mathrm{z} 304)$, amphetamine $(\mathrm{m} / \mathrm{z} 136)$, and norketamine $(\mathrm{m} / z$ 224). To further confirm the identities of the drugs, tandem mass spectrometric analysis was performed to acquire the MS/MS spectra of each drug. Figure $\mathrm{S} 1$ shows the tandem mass spectrometric results. The predominant products derived from each molecular ion were: $\mathrm{m} / \mathrm{z} \quad 313 \rightarrow 266$ (FM2), $\mathrm{m} / \mathrm{z} \quad 324 \rightarrow 223$ (LSD), $\mathrm{m} / \mathrm{z} \quad 194 \rightarrow 163$ (MDMA), $m / z 238 \rightarrow 220$ (ketamine), $m / z \quad 304 \rightarrow 182$ (cocaine), $\mathrm{m} / z \quad 136 \rightarrow 119$ (amphetamine), and $\mathrm{m} / z$ 224 $\rightarrow 207$ (norketamine). The results of the TD-ESI/MS and TD-ESI/MS/MS drug analyses are summarized in Table 1 . The drugs and their product ions are also confirmed via comparison of the mass spectra obtained in this study with those from relevant references. ${ }^{18)}$

According to the College of American Pathologists (CAP), point-of-care tests are designed for use at or near sites where patients are located that do not require permanent dedicated space and are performed outside the physical facilities of clinical laboratories. The turnaround time of a point-of-care test is generally expected to be within $15 \mathrm{~min}$. In this study, the high efficiency of using TD-ESI/MS for

Table 1. Results of MS and MS/MS analyses of six abused drugs and one of their metabolites. The substances were spiked in diluted drained gastric lavage fluid and EA extracted whole blood.

\begin{tabular}{|c|c|c|c|}
\hline Type & Analyte & $\begin{array}{l}\text { Precursor ion } \\
(\mathrm{m} / \mathrm{z})\end{array}$ & $\begin{array}{l}\text { Product ion } \\
\qquad(m / z)\end{array}$ \\
\hline Central nervous & FM2 & 314 & $268^{(\mathrm{b})}, 239$ \\
\hline \multirow[t]{2}{*}{ system depressants } & Ketamine & 238 & $125^{(\mathrm{b})}, 220,207$ \\
\hline & Norketamine $\mathrm{e}^{(\mathrm{a})}$ & 224 & $207^{(\mathrm{b})}, 125$ \\
\hline Central nervous & Cocaine & 304 & $182^{(\mathrm{b})}$ \\
\hline \multirow[t]{2}{*}{ system stimulants } & MDMA & 194 & $163^{(b)}, 105$ \\
\hline & Amphetamine & 136 & $91^{(\mathrm{b})}, 119$ \\
\hline Hallucinogen & LSD & 324 & $223^{(\mathrm{b})}, 281$ \\
\hline
\end{tabular}

\footnotetext{
(a) The main metabolite of ketamine.

(b) Major product ion for mass spectrometric quantitative analysis.
}

drug characterization was demonstrated through a typical analysis taking no more than $30 \mathrm{~s}$. The time required for each analytical process includes: (1) using a metallic probe for sampling $(<5 s),(2)$ thermal desorption of analytes on the probe in the TD-ESI source $(<10 \mathrm{~s})$, (3) electrospray ionization and mass spectrometric detection of the desorbed analytes $(<10 \mathrm{~s})$, and (4) removing residual sample on the probe by burning it away with a high-temperature flame $(<5 s)$.

To study the interferences from the drained gastric lavage fluid specimens, the gastric juice was diluted to 1000 times with normal saline to mimic clinical practices before it was analyzed by TD-ESI/MS. Figures 3(a) and (b) show the mass spectra of the drained gastric lavage fluid specimens collected from two volunteers who received routine gastroendoscopic examinations. As can be seen, the mass spectra recorded from different compounds are very similar. Only a few ion signals were detected, including $\mathrm{m} / \mathrm{z} 135,175,235$, and 257, and no obvious metabolite, peptide, and protein ions were detected. This leaves plenty of space for the mass spectrum to record the drug ion peaks. Figure 3(c) shows the TD-ESI mass spectrum of the diluted gastric juice when spiked with three different abused drugs (including FM2, LSD, and MDMA; 1 ppm each). As it can be seen, all three drugs were detected $(\mathrm{m} / z 314$ for FM2, $m / z 324$ for LSD, and $\mathrm{m} / z 194$ for MDMA) with nearly no interference from the innate biological compounds in the drained gastric lavage fluid. Since ingestion of a combination of drugs is not uncommon, accurate identification of the ingested multiple drugs is also crucial for emergency response.

Due to its high-abundance of metabolites, lipids, peptides, and proteins, the ions on the TD-ESI mass spectrum of human whole blood samples are complex, as shown in Fig. 4(a). This limits the detection of trace chemical compounds such as drugs in whole blood. Figure 4(b) shows the TD-ESI mass spectrum recorded from the whole blood spiked with drugs including ketamine, cocaine, amphetamine, and norketamine (1 ppm each). No drug ion signals were detected, due to the ion suppression effect by innate compounds in whole blood. To overcome the problem, an analytical approach must be developed to efficiently detect drugs with TD-ESI/MS from the whole blood samples.

Liquid-liquid extraction (LLE) is an effective sample pretreatment method used in chemical analysis and has potential for extracting trace drugs in whole blood samples. Figure 5(a) shows the TD-ESI mass spectrum of whole blood extracted with ethyl acetate (EA). It is worth noting that the ion signals detected on the mass spectrum were quite simple and had almost no interference from innate biological compounds in whole blood except for cholesterol ions $(\mathrm{m} / z$ 369). Figure 5(b) shows the TD-ESI mass spectrum after extracting spiked drugs ( 1 ppm each) in the whole blood using EA. The signals of all spiked drugs were successfully detected. The results also show that the interferences from innate compounds in the whole blood could be efficiently removed by organic solvent extraction.

The optimal organic solvent for extracting drugs in blood was examined. Figure 6(a) shows the intensity of drug ions detected in whole blood using different organic solvents for extraction, including dichloromethane (DCM), hexane, toluene and EA. The strongest drug ion signals were obtained by using EA as the extracting solvent; it was then selected to extract drugs in whole blood throughout the study. The 

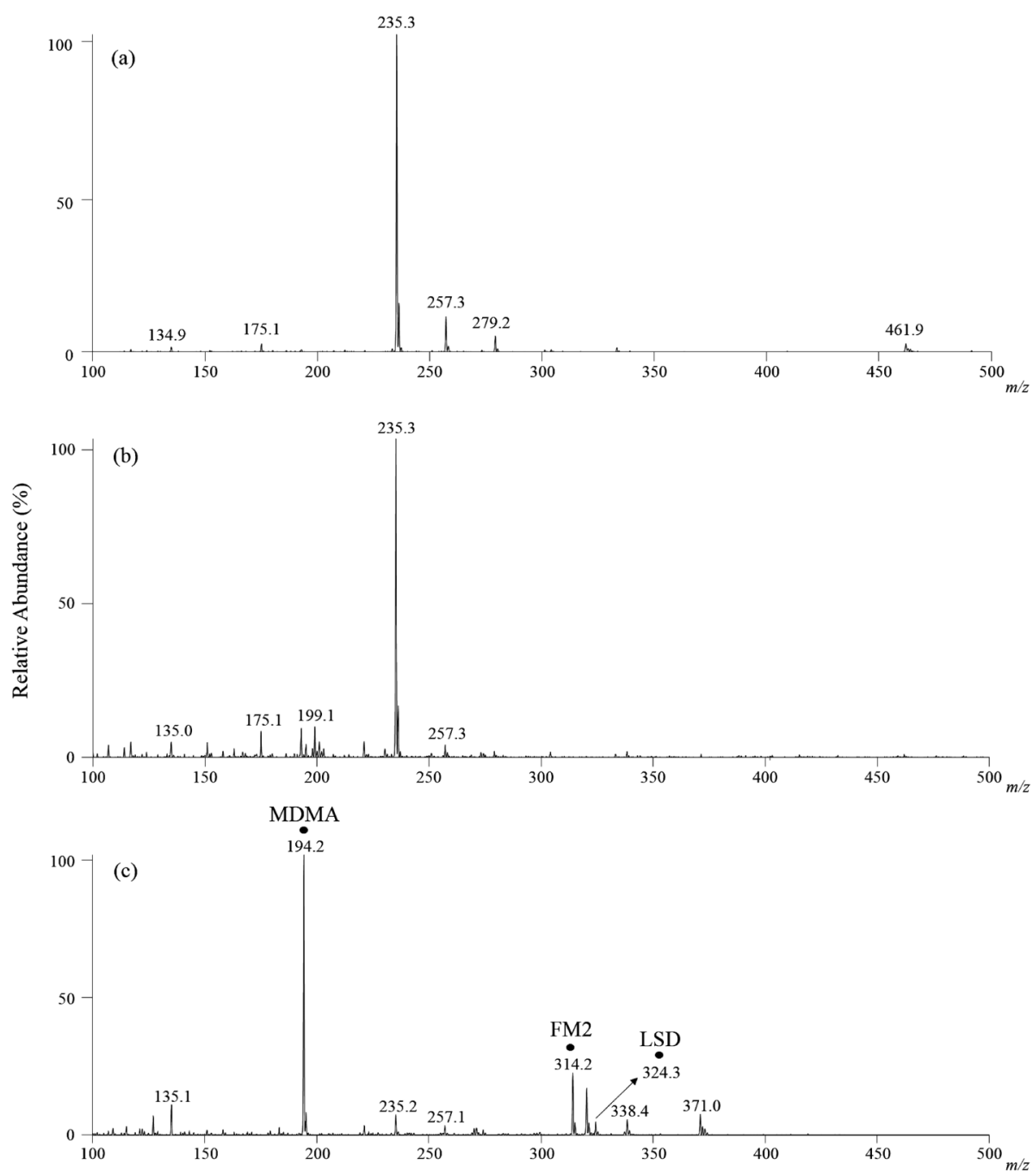

Fig. 3. Positive TD-ESI mass spectra of ( $(a, b)$ diluted gastric juice (diluted for 1000 times with normal saline) collected from two subjects, and (c) diluted gastric juice spiked with FM2, LSD, and MDMA (1 ppm each). drug ion peaks.

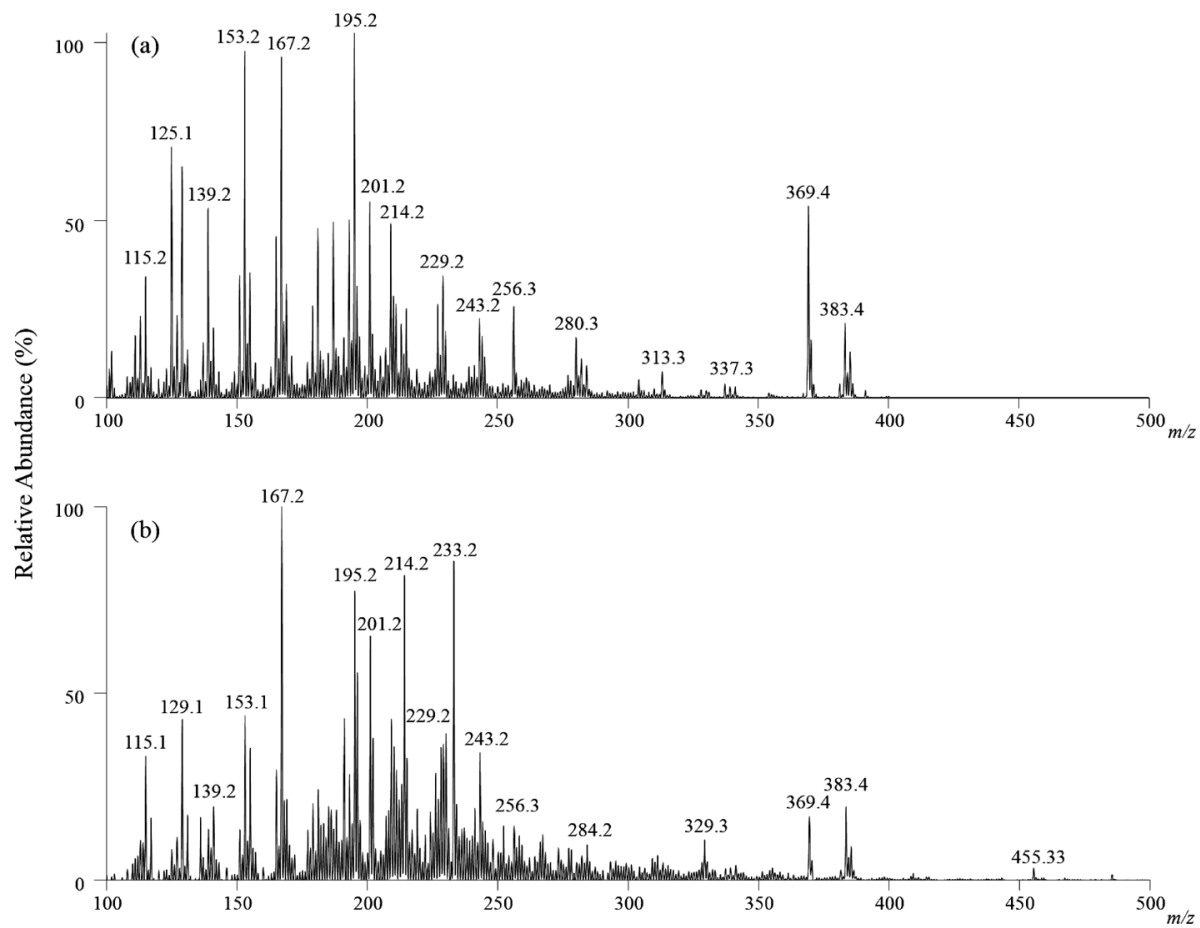

Fig. 4. Positive TD-ESI mass spectra of (a) whole blood specimens, and (b) whole blood spiked with ketamine, cocaine, amphetamine, and norketamine (1 ppm each). : drug ion peaks. 


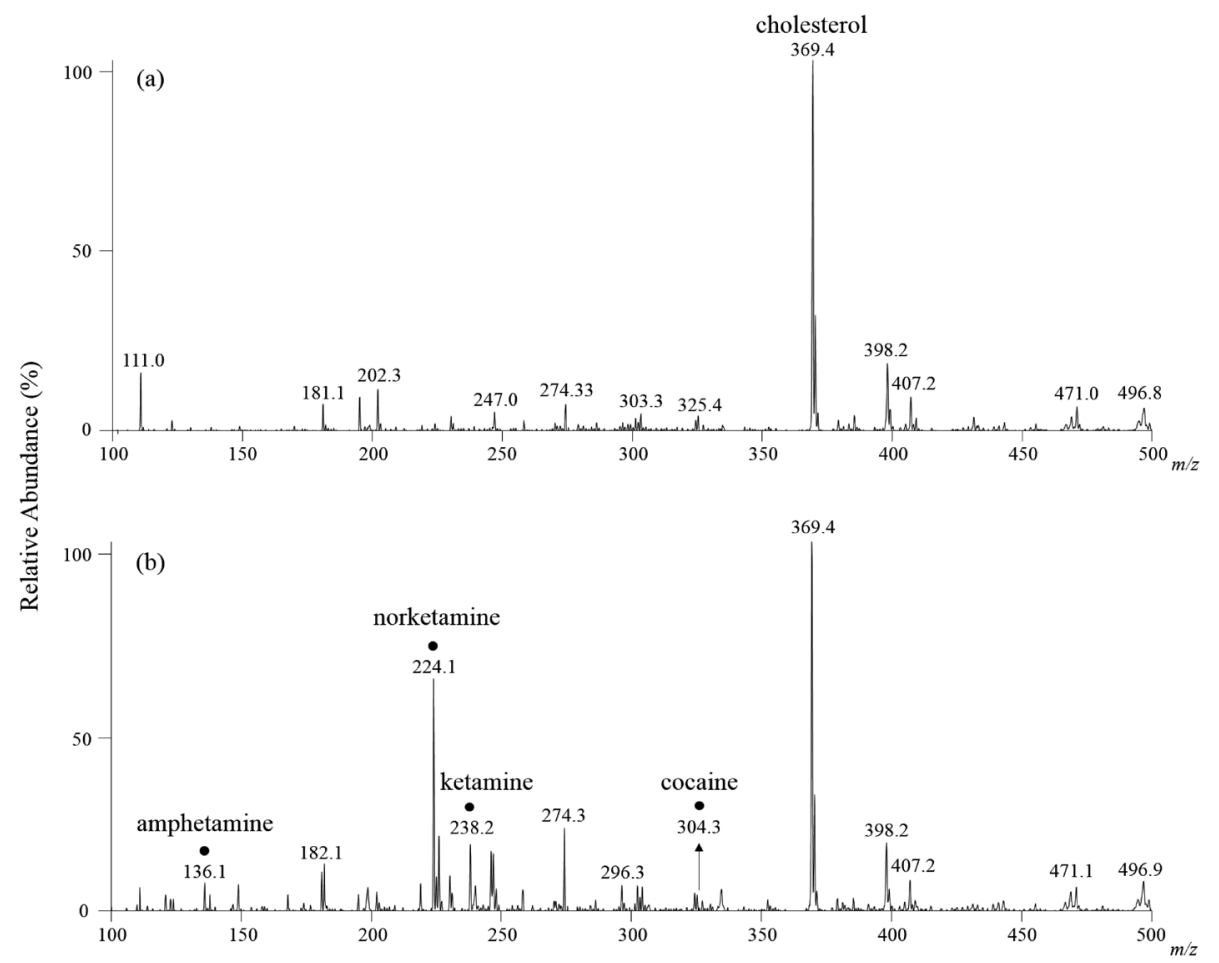

Fig. 5. Positive TD-ESI mass spectra of (a) whole blood specimens extracted with EA, and (b) whole blood spiked with ketamine, cocaine, amphetamine, and norketamine (1 ppm each). : drug ion peaks.

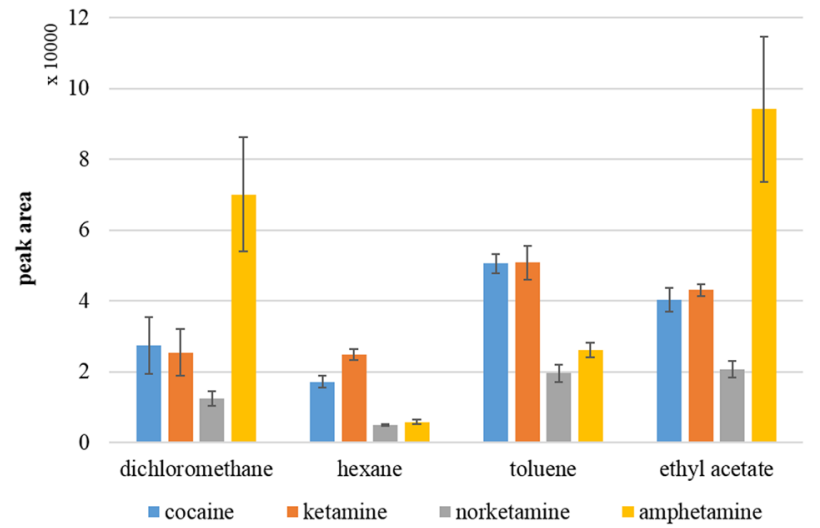

(b)

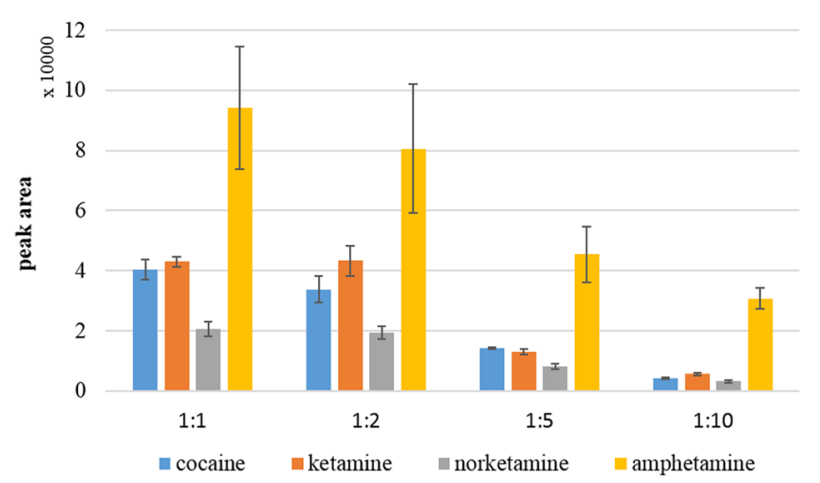

Fig. 6. The intensity of mass spectrometric response of drugs in whole blood with (a) different organic solvents and (b) different blood/EA volume ratios $(1: 1,1: 2,1: 5$, and $1: 10, \mathrm{v} / \mathrm{v})$. (a) MDMA spiked in diluted gastric juice

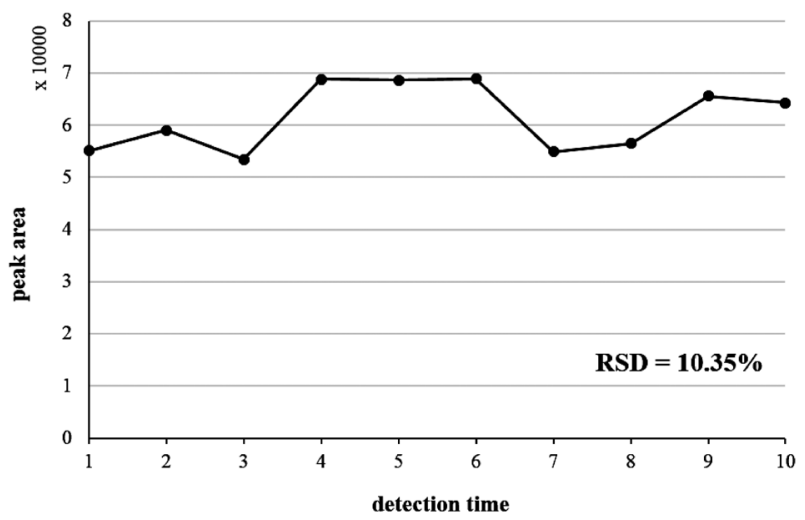

(b) amphetamine spiked in EA extracted whole blood

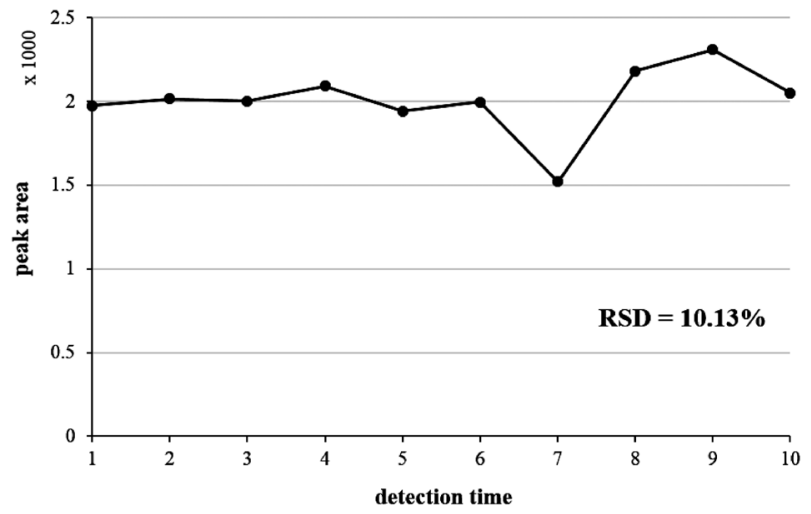

Fig. 7. Repeatability test (10 times) for (a) MDMA (0.5 ppm) spiked in diluted gastric juice and (b) amphetamine (0.5 ppm) spiked in EA extracted whole blood. The drug ions were detected by using TD-ESI/MS in MRM mode. 
volume of EA for extraction was also evaluated. The results presented on Fig. 6(b) show the strongest drug ion signals were obtained by using blood/EA at a volume ratio of $1: 1$.

The stabilities of drug ion signals, detected by TD-ESI/MS in the diluted gastric juice spiked with MDMA (0.5 ppm), were analyzed. The sample solution was repeatedly analyzed by TD-ESI/MS, and Fig. 7 shows the variation of analyte ion signals for 10 repeated analyses. The relative standard deviation (RSD) of the stability test was $10.35 \%$. Similar tests were performed on whole blood, with the samples spiked with amphetamine $(0.5 \mathrm{ppm})$ and extracted with EA. The RSD of the repeated whole blood tests was $10.13 \%$.

To determine the sensitivity of the use of TD-ESI/MS

Table 2. Limit-of-detections (LODs) of abused drugs in diluted drained gastric lavage fluid (subjects 1 and 2) and EA extracted whole blood (subjects 3 and 4 ).

\begin{tabular}{rcccc}
\hline Analyte & FM2 & LSD & MDMA & \\
\hline Subject 1 & $500 \mathrm{ppb}$ & $500 \mathrm{ppb}$ & $1 \mathrm{ppb}$ & \\
Subject 2 & $500 \mathrm{ppb}$ & $500 \mathrm{ppb}$ & $1 \mathrm{ppb}$ & \\
\hline Analyte & Ketamine & Cocaine & Amphetamine & Norketamine \\
\hline Subject 3 & $1 \mathrm{ppb}$ & $1 \mathrm{ppb}$ & $5 \mathrm{ppb}$ & $10 \mathrm{ppb}$ \\
Subject 4 & $1 \mathrm{ppb}$ & $1 \mathrm{ppb}$ & $5 \mathrm{ppb}$ & $10 \mathrm{ppb}$ \\
\hline
\end{tabular}

All LODs of intra-individuals were determined with triplicate measurements. to detect drugs in gastric juice and blood, the limit-ofdetections (LODs) of drugs were determined by analyzing the samples spiked with different concentrations of drugs. The LOD of drug in the diluted gastric juice was $500 \mathrm{ppb}$ for FM2 and LSD, and $1 \mathrm{ppb}$ for MDMA, while the LOD of the drugs extracted from whole blood was $1 \mathrm{ppb}$ for ketamine and cocaine, $5 \mathrm{ppb}$ for amphetamine, and $10 \mathrm{ppb}$ for norketamine. To determine if biofluid samples collected from different individuals would affect the LOD of drugs, the gastric juice and whole blood collected respectively from two male and two female donors were used for analysis. The results showed no difference in LODs of drugs spiked in different individuals (Table 2). The toxic blood levels and/ or the lethal blood levels of six out of the seven drugs tested (documented data of norketamine are not available) in this study are shown in Table S1. These documented clinical data justify the aforementioned fact that LOD of drugs in drained gastric lavage fluid and whole blood by TD-ESI/MS is at sub ppm level, which is sensitive enough for emergency medical application, since the quantities of drugs ingested by substance abusers are usually much higher than LOD, except FM2 and LSD (due to their relatively higher boiling point). The response of the drug ion signals recorded from the diluted gastric juice and EA extracted whole blood was studied. As shown in Fig. 8, linear responses for all drugs with concentrations ranging between $5 \mathrm{ppb}$ and $1 \mathrm{ppm}$ range were obtained with a $R^{2}$ between 0.9887 and 0.9995 .
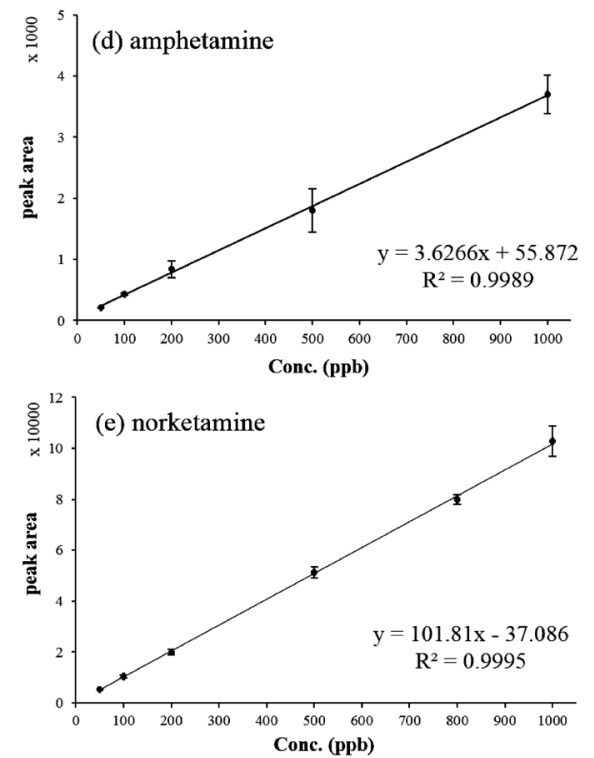
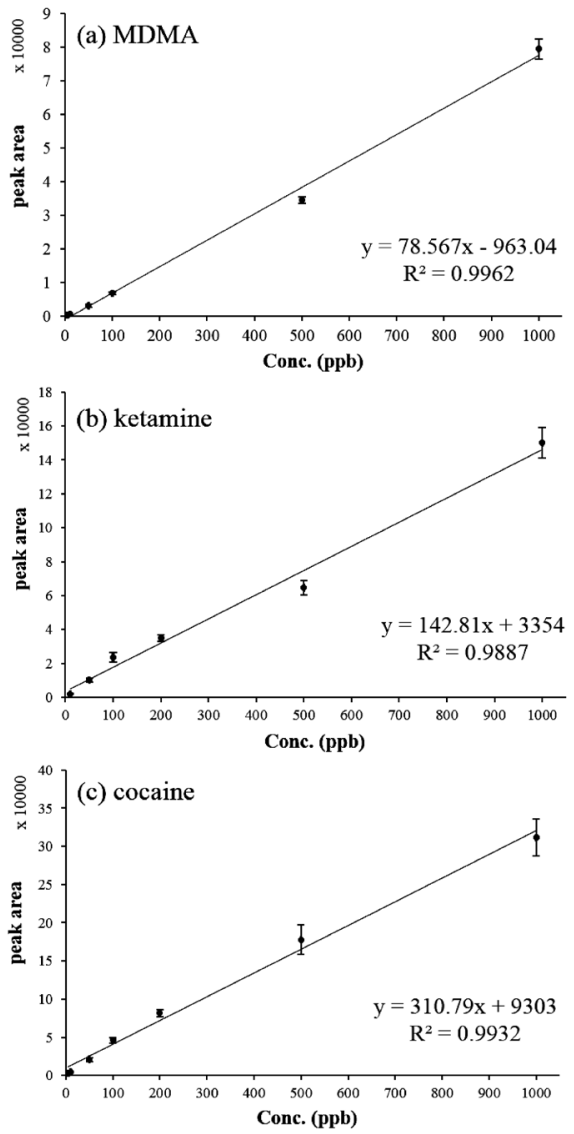

Fig. 8. Calibration curves of (a) MDMA spiked in diluted gastric juice, and (b) ketamine, (c) cocaine, (d) amphetamine, and (e) norketamine spiked in EA extracted whole blood. The concentrations of the drugs varied from $5 \mathrm{ppb}$ to $1 \mathrm{ppm}$, and the ion signals were detected by TDESI/MS in MRM mode. 


\section{CONCLUSION}

In contrast to the traditional GC/MS and LC/MS that require time and labor-consuming sample pretreatment, TD-ESI/MS provides an efficient diagnostic strategy for rapidly characterizing drugs in drained gastric lavage fluid and whole blood extract. The technique's high efficiency justifies its use as a point-of-care diagnostic tool in emergency settings. The LOD of drugs in drained gastric lavage fluid and whole blood by TD-ESI/MS is at sub ppm level, which is sensitive enough for emergency medical application, since the quantities of drugs ingested by substance abusers are usually much higher than LOD. In addition, the technique is suitable for accurate quantification and exhibits high repeatability on drug analysis. Once the mass spectrum of an abused drug is obtained by means of the TD-ESI/MS approach, the data can be compared with mass spectral databases for efficient matching and compound identification.

\section{Acknowledgements}

This work was supported partially by the NSYSU-KMU JOINT RESEARCH PROJECT (\#NSYSUKMU 105-I006) and the Ministry of Science and Technology of Taiwan (\#04B393322).

\section{REFERENCES}

1) National Drug Intelligence Center. (2011). The Economic Impact of Illicit Drug Use on American Society. Washington D.C.: United States Department of Justice. http://www.justice.gov/archive/ndic/ pubs44/44731/44731p.pdf (PDF, 2.4MB)

2) G. W. Fulde, S. L. Forster. The impact of amphetamine-type stimulants on emergency services. Curr. Opin. Psychiatry 28: 275-279, 2015.

3) K. F. Hawk, F. E. Vaca, G. D'Onofrio. Reducing fatal opioid overdose: Prevention, treatment and harm reduction strategies. Yale J. Biol. Med. 88: 235-245, 2015.

4) B. Ramoo, M. Funke, C. Frazee, U. Garg. Comprehensive urine drug screen by gas chromatography/mass spectrometry (GC/MS). Methods Mol. Biol. 1383: 125-131, 2016.

5) W. M. Bosker, M. A. Huestis. Oral fluid testing for drugs of abuse. Clin. Chem. 55: 1910-1931, 2009.

6) Y. W. Lee. Simultaneous screening of 177 drugs of abuse in urine using ultra-performance liquid chromatography with tandem mass spectrometry in drug-intoxicated patients. Clin. Psychopharmacol. Neurosci. 11: 158-164, 2013.

7) M. Z. Huang, C. C. Zhou, D. L. Liu, S. S. Jhang, S. C. Cheng, J.
Shiea. Rapid characterization of chemical compounds in liquid and solid states using thermal desorption electrospray ionization mass spectrometry. Anal. Chem. 85: 8956-8963, 2013.

8) C. Shiea, Y. L. Huang, D. L. Liu, C. C. Chou, J. H. Chou, P. Y. Chen, J. Shiea, M. Z. Huang. Rapid screening of residual pesticides on fruits and vegetables using thermal desorption electrospray ionization mass spectrometry. Rapid Commun. Mass Spectrom. 29: 163-170, 2015.

9) Z. Takáts, J. M. Wiseman, B. Gologan, R. G. Cooks. Mass spectrometry sampling under ambient conditions with desorption electrospray ionization. Science 306: 471-473, 2004

10) Z. Takáts, J. M. Wiseman, R. G. Cooks. Ambient mass spectrometry using desorption electrospray ionization (DESI): Instrumentation, mechanisms and applications in forensics, chemistry, and biology. J. Mass Spectrom. 40: 1261-1275, 2005.

11) J. F. García-Reyes, A. U. Jackson, A. Molina-Díaz, R. G. Cooks. Desorption electrospray ionization mass spectrometry for trace analysis of agrochemicals in food. Anal. Chem. 81: 820-829, 2009.

12) R. B. Cody, J. A. Laramée, H. D. Durst. Versatile new ion source for the analysis of materials in open air under ambient conditions. Anal. Chem. 77: 2297-2302, 2005.

13) S. E. Edison, L. A. Lin, B. M. Gamble, J. Wong, K. Zhang. Surface swabbing technique for the rapid screening for pesticides using ambient pressure desorption ionization with high-resolution mass spectrometry. Rapid Commun. Mass Spectrom. 25: 127-139, 2011.

14) J. Shiea, M. Z. Huang, H. J. Hsu, C. Y. Lee, C. H. Yuan, I. Beech, J. Sunner. Electrospray-assisted laser desorption/ionization mass spectrometry for direct ambient analysis of solids. Rapid Commun. Mass Spectrom. 19: 3701-3704, 2005.

15) C. W. Lee, H. Su, P. Y. Chen, S. J. Lin, J. Shiea, S. J. Shin, B. H. Chen. Rapid identification of pesticides in human oral fluid for emergency management by thermal desorption electrospray ionization/mass spectrometry. J. Mass Spectrom. 51: 97-104, 2016.

16) C. W. Lee, H. Su, K. D. Wu, J. Shiea, D. C. Wu, B. H. Chen, S. J. Shin. Rapid point-of-care identification of oral medications in gastric lavage content by ambient mass spectrometry in emergency room. Rapid Commun. Mass Spectrom. 30: 1295-1303, 2016.

17) M. E. Kleinman, L. Chameides, S. M. Schexnayder, R. A. Samson, M. F. Hazinski, D. L. Atkins, M. D. Berg, A. R. de Caen, E. L. Fink, E. B. Freid, R. W. Hickey, B. S. Marino, V. M. Nadkarni, L. T. Proctor, F. A. Qureshi, K. Sartorelli, A. Topjian, E. W. van der Jagt, A. L. Zaritsky. Part 14: pediatric advanced life support: 2010 American Heart Association Guidelines for Cardiopulmonary Resuscitation and Emergency Cardiovascular Care. Circulation 122(Suppl. 3): S876-S908, 2010.

18) H. H. Lee, J. F. Lee, S. Y. Lin, B. H. Chen. Simultaneous identification of abused drugs, benzodiazepines, and new psychoactive substances in urine by liquid chromatography tandem mass spectrometry. Kaohsiung J. Med. Sci. 32: 118-127, 2016. 